\title{
The use of discrete orthogonal projections in boundary element methods
}

\author{
J.H. Brandts *
}

\begin{abstract}
In recent papers by Sloan and Wendland (1999), Grigorieff and Sloan (1998) and Grigorieff, Sloan and Brandts (2000), a formalism was developed that serves many important and interesting applications in boundary element methods: the "commutator property" for splines. Based on superapproximation results, this property is, for example, a tool of central importance in stability and convergence proofs for qualocation methods for boundary integral equations with variable coefficients.

Another application is the transfer of superconvergence properties from constant-coefficient boundary integral equations to the variable coefficient case. The heart of the theory is formed by the concept "discrete orthogonal projection", that arises when the $L^{2}$-orthogonal inner product is discretized by possibly non-standard quadrature rules.

In this paper, we present an overview of the theory of discrete orthogonal projections, and a new set of numerical experiments that confirm the theory. The main conclusion is that the presence of variable coefficients of a certain smoothness does not influence superconvergence in a negative way, and that henceforth the use of superconvergence-based a posteriori error estimators in this particular case is theoretically justified.
\end{abstract}

\section{Introduction}

This paper aims to summarize recent results in the area of discretization methods for boundary integral equations. Those results were obtained by the use of a theory that involves the concept of so-called discrete orthogonal projections. As we will show, discrete orthogonal projections provide a connecting framework between the left-bank of collocation methods and the right-bank of Galerkin boundary element discretizations. We will, however, mainly concentrate on the massive river of qualocation methods, which is situated in between.

\subsection{Stability in $L_{p}$ of discrete orthogonal projections}

Writing $R_{h}$ for a discretized version (to be specified in detail later on) of the $L_{2}$ orthogonal projection $P_{h}$ on some spline finite element space $S_{h}$, we ask (and try to answer) questions of the following nature. Firstly, under which conditions is discrete

*Mathematics Institute, Utrecht University, Budapestlaan 6, 3584 CD Utrecht, The Netherlands. 
orthogonal projection $p$-stable, in the sense that, denoting the space of continuous functions on the unit interval $I$ by $C(I)$,

$$
\forall f \in C(I), \quad\left\|R_{h} f\right\|_{p} \leq C|f|_{h, p}
$$

for some corresponding discrete version $|\cdot|_{h, p}$ of the $L_{p}$-norm on $C(I)$. Since the $L_{p}$-stability of the projection $P_{h}$ has already been established, see for example De Boor [1] and Crouzeix and Thomée [2], one could try to adapt their proofs to the discretized situation. Indeed, for continuous splines on arbitrary meshes this was done in the paper [4] by Grigorieff and Sloan. Also, approximation properties and the case of zero boundary conditions were considered in that paper.

For the important practical case of periodic boundary conditions, which corresponds to the situation of functions living on a closed curve, refinements of the stability theorems appeared necessary. The analysis leading to these refinements was given by Grigorieff, Sloan and Brandts in Section 5 of [5].

\subsection{Superapproximation, localization principle}

Once the $p$-stability was established, more sophisticated questions could be posed. In Prössdorf's paper [7] it was remarked that many finite element spaces have the property that

$$
\left\|\left(I-P_{h}\right) G P_{h}\right\|_{p} \rightarrow 0, \text { for } h \rightarrow 0,
$$

where $G: f \mapsto g f$ stands for multiplication with a sufficiently smooth fixed function g. A simple but important consequence of this property is the convergence in $L_{p}$ of the underlying finite element method for the discretization of $G$. One could ask if (2) also holds in the discrete sense, i.e. with $P_{h}$ replaced by $R_{h}$. This question too has been answered affirmatively. In the case of periodic smoothest splines on uniform grids it follows from a much stronger result by Sloan and Wendland in [9], which involves a scale of Sobolev norms and a pseudo-differential operator $L$. In the case $L=I$, their result reduces to a statement about $\left(I-P_{h}\right) G P_{h}$. For periodic and non-periodic continuous splines on non-uniform meshes, results in the spirit of (2) can be found in [5]. Contrary to [9], this paper contains only results in the $L_{p}$ and $W_{p}^{1}$ norms.

Remark 1.1 As one may expect, the periodic smoothest splines case in [9] was tackled using a technically complicated Fourier analysis. This line of proof was successfully abandoned in [5] in order to be able to include non-uniform grids, which left the question whether it would be possible to reformulate the periodic smoothest splines case without the Fourier analysis and perhaps obtain results for less restricted meshes as well.

\subsection{Commutator property}

In the non-discrete case, it was fairly easy to obtain results for the dual operator $P_{h} G\left(I-P_{h}\right)$ using duality between $L_{p}$ spaces. In the discrete case, however, one had to reconsider the basic duality, since $R_{h}$ is, contrary to $P_{h}$, not by default $L_{2}$ self-adjoint. Once this problem was solved, the equality

$$
\left[G, R_{h}\right]:=G R_{h}-R_{h} G=\left(I-R_{h}\right) G R_{h}-R_{h} G\left(I-R_{h}\right)
$$


combined the results of the two into one single statement, which is referred to as the "commutator property", while the results for the two separate parts is often referred to as "superapproximation property" or "localization principle". The commutator property for the different settings can be found in the papers [9] and [5]. Again, the results in [9] are formulated in a whole scale of Sobolev norms and involve a pseudo-differential operator $L$, while in [5] the analysis is restricted to $L_{p}$ and $W_{p}^{1}$.

\subsection{Outline of this paper}

We will start with defining discrete orthogonal projections in Section 2 and recalling as well as illustrating some of their basic properties. We also consider the relatively simple case of discrete projections on discontinuous splines. As far as we know. this case was not studied in the literature, even though it could have implications for fully discrete Galerkin methods for two-point boundary value problems. In Section 3 we concentrate on two applications of the commutator property, which show how to deal with variable coefficients. It should be stressed that, even though the concept is easy, it should not be underestimated that all results hold for non-uniform grids, which means they are mathematically sound also in the case of adaptive grid refinement.We also provide some numerical experiments that illustrate some subtleties of the stability theory of [5] in the context of the discretization of the operator $G$. Finally, in Section 5, we reflect on future research.

\section{Discrete orthogonal projections}

Discrete projections and inner products on spline spaces are defined using composite non-standard quadrature rules relative to a given arbitrary partitioning. We refer to [4] and [5] for more details on the continuous splines case. See also [3] and [6] for related topics.

\subsection{Spline spaces and partitioning}

Let $\pi_{h}:=\left\{0=x_{0}<x_{1}<\cdots<x_{n}=1\right\}$ be a partitioning of the unit interval $I$. Denote the space of continuous functions on $I$ by $C^{0}(I)$, and the space of functions that are piecewise continuous relative to $\pi_{h}$ by $C^{-1}(I)$. Define for each $r \geq 1$ and $m \in\{-1,0\}$ the spline-space $S_{h}^{m, r}$ of (dis-)continuous piecewise polynomials of degree less than or equal to $r-1$ relative to $\pi_{h}$, by

$$
S_{h}^{m, r}:=\left\{\psi \in C^{m}(I):\left.\psi\right|_{I_{k}} \in P_{r-1}\left(I_{k}\right), \quad k=0, \cdots, n-1\right\},
$$

where $P_{d}(\cdot)$ is the space of polynomials of degree less than or equal to $d$ and $I_{k}:=$ $\left[x_{k}, x_{k+1}\right]$ for $k \in\{0, \cdots, n-1\}$. We will proceed to define discrete inner products on the spaces $S_{h}^{m, r}$, distinguishing between $m=-1$ and $m=0$.

\subsection{Composite quadrature rules relative to $\pi_{h}$}

First, define a $J$-point quadrature rule $Q$ on $C^{0}(I)$ by

$$
Q g:=\sum_{j=1}^{J} w_{j} g\left(\xi_{j}\right) \sim \int_{0}^{1} g(x) d x,
$$


where the weights $w_{j}$ are positive and the sample-points $\xi_{j}$ are strictly increasing in the unit interval. Transforming this quadrature rule to each of the subintervals $I_{k}$ defined by the partitioning $\pi_{h}$ gives rise to a composite rule on $C^{-1}(I)$ as follows,

$$
Q_{h} g:=\sum_{k=0}^{n-1} h_{k} \sum_{j=1}^{J} w_{j} g\left(x_{k, j}\right) \sim \int_{0}^{1} g(x) d x
$$

where $h_{k}:=x_{k+1}-x_{k}$ denotes the length of $I_{k}$, and $x_{k, j}:=x_{k}+h_{k} \xi_{j}$ is the position of the $j$-th sample point in that sub-interval. We should use the quadrature rule with care if $g$ has discontinuities at the partitioning points: if $x_{k, j}$ is a left (right) boundary point of a subinterval, then $g\left(x_{k, j}\right)$ should be understood as the limiting value of $g(x)$ coming from the right (left). One can easily check that

$$
(f, g)_{h}:=Q_{h}(f \bar{g}), \quad f, g \in C(I),
$$

is a positive semi-definite Hermitian sesqui-linear form. We will formulate conditions under which it gives rise to an inner product on the spline spaces.

\subsection{Discrete inner products}

In [4] it was shown that $(\cdot, \cdot)_{h}$ is an inner product on $S_{h}^{0, r}$ if and only if $J \geq r \geq 2$. An argument similar (but easier) to that in [4] shows that the same holds for $S_{h}^{-1, r}$.

Lemma 2.1 The form $(\cdot, \cdot)_{h}$ is an inner product on $S_{h}^{-1, r}$ if and only if $J \geq r \geq 1$.

Proof. First, suppose $J \geq r \geq 1$. The only thing we need to show is that

$$
\forall \psi \in S_{h}^{-1, r}, \quad(\psi, \psi)_{h}=0 \Leftrightarrow \psi=0 .
$$

Since $(\psi, \phi)_{h}=0$ if $\psi$ and $\phi$ have disjoint supports, it is sufficient to show property (8) only locally on each subinterval, which translates to

$$
\forall q \in P_{r-1}(I), \quad Q\left(|q|^{2}\right)=0 \Leftrightarrow q=0 .
$$

But this follows from the fact that if $Q\left(|q|^{2}\right)=0$, then $q$ has at least $J \geq r$ roots, so $q$ must be zero. The fact that if $J<r$ we do not get an inner product is trivial: there exist $0 \neq q \in P_{r-1}(I)$ zero in all $J$ quadrature points. This proves the statement.

Now, suppose we change the scene to periodic boundary conditions, or, equivalently, to functions on the circle. Then Lemma 2.1 clearly still holds if and only if $J \geq r \geq 1$. In the piecewise continuous case, however, there is a subtle difference, which is due to the loss of one degree of freedom in the discrete space, caused by the condition $\psi_{h}(0)=\psi_{h}(1)$. Writing $C^{\pi}(I)$ and $S_{h}^{\pi}$ for the periodic counterparts of the spaces $C[0, L]$,

$$
C^{\pi}(I):=\{f \in C(I): f(0)=f(1)\}, \quad \text { and } \quad S_{h}^{\pi, r}=S_{h}^{0, r} \cap C^{\pi}(I),
$$

the problem of characterizing $(\cdot, \cdot)_{h}$ as an inner product on $S_{h}^{\pi, r}$ was solved in Section 5 of [5] as follows. Let $\phi$ be the polynomial of degree $J$ defined by

$$
\phi(\xi):=\prod_{j=1}^{J}\left(\xi-\xi_{j}\right),
$$


so it is the unique monomial that has its zeros in the quadrature points. The following characterization is taken from [5].

Proposition 2.2 The positive semidefinite Hermitian sesqui-linear form $(\cdot, \cdot)_{h}$ is an inner product on $S_{h}^{\pi, r}$ if and only if either $J \geq r$, or $J=r-1$ and one of the following conditions is satisfied:

(1) $|\phi(0)| \neq|\phi(1)|$

(2) $\phi(0)=-\phi(1) \neq 0$ and $n$ is odd.

As an illustration of which extra inner products the case $J=r-1$ generates, consider the space $S_{h}^{\pi, 2}$ where $\pi_{h}$ is a uniform partition of the circle into $n$ subintervals. Let $\xi_{1}=0.5$. If $n$ is even, it is easy to construct a function $0 \neq \psi_{h} \in S_{h}^{\pi, 2}$ such that $\left(\psi_{h}, \psi_{h}\right)_{h}=0$, namely, the continuous piecewise linear defined by the nodal values $\psi\left(x_{i}\right)=(-1)^{i}$ (see Figure 1, left). Note that, in particular, $\psi_{h}(0)=\psi_{h}(1)$. If $n$ is odd, however, the same construction shows that it is impossible to construct a non-trivial $\psi_{h} \in S_{h}^{\pi, r}$ that is zero on all quadrature points.

$n$ EVEN, J=r-1=1, xi=0.5, NO INNER PRODUCT

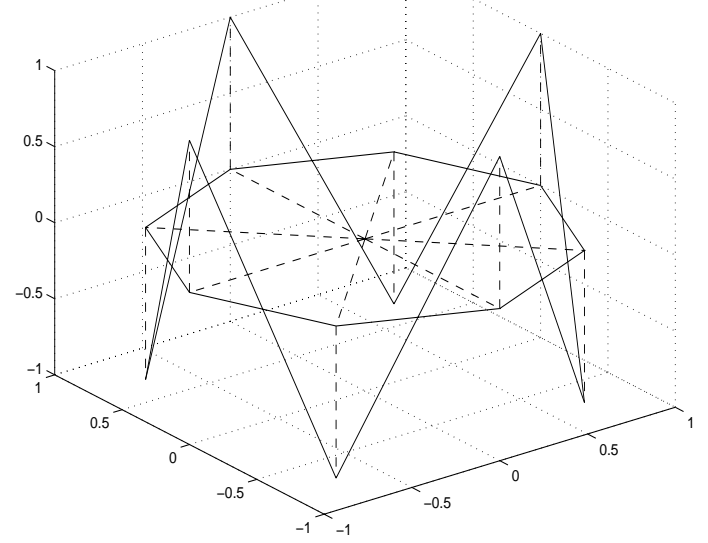

EVEN, J=r-1=1, WELL-DEFINED INNER PRODUCT

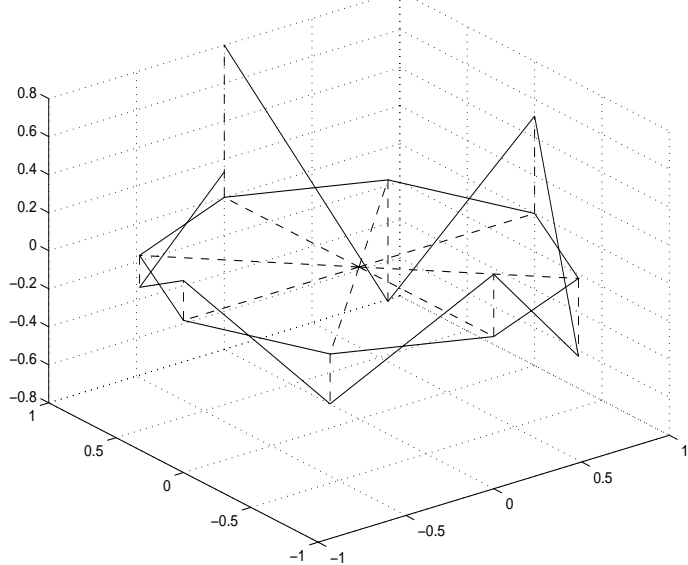

Figure 1. Illustration to Proposition 2.2.

In general, if $J=r-1$ and condition (1) of Proposition 2.2 is satisfied, we may assume, without loss of generality, that $|\phi(0)|<|\phi(1)|$. Continuous gluing together an arbitrary amount of copies of $\phi$ (transformed to each subinterval) results in strictly increasing nodal values, so the resulting function can never be periodic and continuous (see Figure 1, right). Note that this holds even if the partitioning is non-uniform, and regardless of the number of subintervals.

\subsection{Discrete orthogonal projections}

From well-defined discrete inner products one can derive in a consistent way discrete orthogonal projections $R_{h}^{m, r}: C^{m}(I) \rightarrow S_{h}^{m, r}$ as follows. For convenience, we will suppress the superscripts $m$ and $r$ unless they are relevant.

$$
R_{h} f \in S_{h}, \quad\left(R_{h} f, \psi_{h}\right)_{h}=\left(f, \psi_{h}\right)_{h} \text { for all } \psi_{h} \in S_{h} .
$$


From this definition it might become intuitively clear that $R_{h} f$ can be a good approximation to $f$ even for non-accurate (possibly even divergent) quadrature rules, since anything the rule "does wrong", it does so on both sides of the equality sign. To study the approximation quality of a family $\left\{R_{h}\right\}$ of discrete orthogonal projections, we define the semi-norms $|\cdot|_{h, p}$ on $C^{-1}(I)$, as discrete counterparts of the p-norms $\|\cdot\|_{p}$ on $L_{p}(I)$ as follows,

$$
|f|_{h, p}:=Q_{h}\left(|f|^{p}\right)^{1 / p} \quad \text { for } 1 \leq p<\infty, \quad \text { and } \quad|f|_{h, \infty}:=\max _{k, j}\left\{\left|f\left(x_{k, j}\right)\right|\right\} .
$$

Based on the fact that if $J \geq r \geq 2$, the mapping $\phi \mapsto \sqrt{|\phi|^{2}}$ defines a norm on $P_{r-1}(I)$, it was proved in [5] that $|\cdot|_{h, p}$ and $\|\cdot\|_{p}$ are equivalent on $S_{h}^{0, r}$. The same argument can in fact be used to prove that this equivalence also holds on $S_{h}^{-1, r}$ if $J \geq r \geq 1$, where again it does not matter if the periodic or non-periodic case is considered. Clearly, $\phi \mapsto \sqrt{|\phi|^{2}}$ does not define a norm on $P_{r-1}(I)$ if $J \leq r-1$, and equivalence on $S_{h}^{\pi, r}$ (if present) should be proved differently. That the equivalence does not hold for the inner products generated by case (2) of Lemma 2.1 proves the following counter-example.

Proposition 2.3 If the norm $|\cdot|_{h, p}$ is generated by an inner product satisfying case (2) of Lemma 2.1, it is not equivalent to the norm $\|\cdot\|_{p}$.

Proof. Consider the piecewise linear case, with a quadrature rule defined by a weight $w=1$ and a quadrature point $\xi=\frac{1}{2}$. Given a partition $\pi_{h}$ into an odd number $n$ of sub-intervals, define $\psi_{h} \in S_{h}^{\pi}$ by

$$
\psi_{h}\left(x_{j}\right)=(-1)^{j}, \quad(j=1, \cdots, n), \quad \psi_{h}\left(x_{0}\right):=(-1)^{n} .
$$

On all intervals except the first, we have defined scaled copies of the function $\phi$ so $\psi_{h}$ is zero at all the quadrature points except for the point $x=\frac{1}{2} h_{0}$. There it has the value -1 and therefore,

$$
\left|\psi_{h}\right|_{h, 2}^{2}=h_{0} .
$$

Concerning the norm $\left\|\psi_{h}\right\|_{2}$, we first note that for a linear function on the interval $[a, b]$ with left- and right boundary values $L$ and $R$,

$$
\int_{a}^{b}\left(\frac{b-x}{b-a} L+\frac{x-a}{b-a} R\right)^{2} d x=\frac{b-a}{3}\left(L^{2}+L R+R^{2}\right) .
$$

This gives us that

$$
3\left|\psi_{h}\right|_{2}^{2}=3 h_{0}+\sum_{j=1}^{n-1} h_{j}=2 h_{0}+1
$$

This presents a counter example for the equivalence of the norms in the case $r=2$. Clearly, a similar construction can be employed to disprove equivalence for other (even) values of $r$.

Definition 2.4 Let $m \in\{0,1, \pi\}$ and let $\left\{R_{h}\right\}$ be a well-defined sequence of discrete orthogonal projections $R_{h}: C^{m}(I) \rightarrow S_{h}^{m, r}$. Then this sequence is called $p$-stable if

$$
\forall f \in C^{m}(I), \quad\left\|R_{h} f\right\|_{p} \leq C|f|_{h, p} .
$$


Sufficient conditions for $p$-stability can be found in [4] and [5]. This includes conditions for the periodic piecewise continuous case (circumventing the technical difficulty that $N$ does not define a norm on $P_{r-1}(I)$ if $\left.J \leq r-1\right)$. For projections on $S_{h}^{-1, r}$, the $p$-stability can be derived from the $p$-stability of projections on $S_{h}^{0, r}$ with the trivial partition $\pi_{h}=\{0,1\}$ and the fact that projection on $S_{h}^{-1, r}$ is a local matter.

In the cases that discrete projections are $p$-stable, they inherit the optimal order approximation properties of the projections $P_{h}: C^{m}(I) \rightarrow S_{h}^{m, r}$ in both the $L_{p}$-norm and its discrete version. This is due to the estimate

$$
\left\|R_{h} f-f\right\|_{p} \leq\left\|R_{h}\left(f-\psi_{h}\right)\right\|_{p}+\left\|f-\psi_{h}\right\|_{p} \leq C\left|f-\psi_{h}\right|_{h, p}+\left\|f-\psi_{h}\right\|_{p} .
$$

which transforms the question of finding a priori $L_{p}$-bounds for $R_{h} f$, into one of approximation theory.

\subsection{The Commutator Property}

In many applications, the following question is of interest. Does it matter if we change the order of the two operations "projection on a spline space" and "multiplication with a smooth function"? First we will discuss a special case. The following fact is well-known, and shows when $R_{h}$ reduces to a collocation operator.

Proposition 2.5 Let $\xi_{0}=0, \xi_{n}=1$ and $J=r$, and $S_{h}=S_{h}^{0, r}$. Then $\left(R_{h} f\right)\left(x_{j, k}\right)=$ $f\left(x_{j, k}\right)$ for all $j$ and $k$, i.e., $R_{h}$ is an interpolation operator. This is also the case if $S=S_{h}^{-1, r}$ and $J=r$.

Proof. Under the given conditions, the space $S_{h}^{m, r}$ has a nodal basis, which means, a basis $\left\{\psi_{j, k}\right\}$ which has the property $\psi_{j, k}\left(x_{u, v}\right)=\delta_{j u} \delta_{k v}$. This leads to

$$
w_{k} R_{h} f\left(x_{j, k}\right)=\left(R_{h} f, \psi_{j, k}\right)_{h}=\left(f, \psi_{j, k}\right)_{h}=w_{k} f\left(x_{j, k}\right),
$$

which proves the statement. In the discontinuous setting and for rules which include one or both boundary points, the proper limiting values of the functions involved should be taken.

In case the discrete projection is an interpolation, clearly $G R_{h}\left(x_{j, k}\right)=R_{h} G\left(x_{j, k}\right)$, where $G$ stands for multiplication with a function $g$. The expectation is that for other quadrature rules, the equality will be lost, but not dramatically, in the sense that the difference will still be small. For continuous splines, this was proved in [5] where it was formulated as follows.

Theorem 2.6 Let $m \in\{0, \pi\}$. Let $p \in[1, \infty]$ and assume that $\left\{R_{h}^{m, r}\right\}$ is $p$-stable and $q$-stable, where $\frac{1}{p}+\frac{1}{q}=1$. Then for all $f \in C^{m}(I)$,

$$
\left|\left(G R_{h}-R_{h} G\right) f\right|_{h, p}+\left\|\left(G R_{h}-R_{h} G\right) f\right\|_{p} \leq C h \|\left. g^{\prime}\right|_{r-1, \infty}|f|_{h, p} .
$$

For the discontinuous setting, we will now formulate and prove a similar result. The proof is based on a Bramble-Hilbert like argument and much less complicated than the proof for Theorem 2.6. 
Theorem 2.7 Let $p \in[1, \infty]$ and assume that $\left\{R_{h}^{-1, r}\right\}$ is $p$-stable. Then for all $f \in C^{-1}(I)$,

$$
\left|\left(G R_{h}-R_{h} G\right) f\right|_{h, p}+\left\|\left(G R_{h}-R_{h} G\right) f\right\|_{p} \leq C h \|\left. g^{\prime}\right|_{0, \infty}|f|_{h, p} .
$$

Proof. First note that, due to the local character of projection on $S_{h}^{-1, r}$, for all $\psi_{h} \in S_{h}^{-1,1}$ we have that $\psi_{h} R_{h}=R_{h} \psi_{h}$. This gives, using $p$-stability twice, that for all $\psi_{h} \in S_{h}^{-1,1}$,

$$
\begin{aligned}
\left\|\left(G R_{h}-R_{h} G\right) f\right\|_{p} & =\left\|\left(G-\psi_{h}\right) R_{h} f-R_{h}\left(G-\psi_{h}\right) f\right\|_{p} \\
& \leq\left\|\left(G-\psi_{h}\right) R_{h} f\right\|_{p}+\left\|R_{h}\left(G-\psi_{h}\right) f\right\|_{p} \\
& \leq\left\|g-\psi_{h}\right\|_{\infty}\left\|R_{h} f\right\|_{p}+C\left|\left(g-\psi_{h}\right) f\right|_{h, p} \\
& \leq C\left(\left\|g-\psi_{h}\right\|_{\infty}+\left|g-\psi_{h}\right|_{h, \infty}\right)|f|_{h, p} .
\end{aligned}
$$

Applying standard approximation theory completes the first part of the proof. The second part is similar, but also uses the equivalence of norms to conclude that $\left|R_{h} f\right|_{h, p} \leq C|| R_{h} f \|_{p} \leq C|f|_{h, p}$

\section{Application of the Commutator Property}

Here we will present two easy examples of situations in which the commutator property can be successfully applied to deal with variable coefficients. For the more realistic applications, we suggest to use the smoothest-spline commutator property in [9] which we did not treat in this paper, but which has more potential due to the formulation in the context of pseudo-differential operators. A direct application can be found in $[10]$ by the same authors.

\subsection{Weighted discrete inner products}

We will show here that a smooth weight function defining a weighted discrete inner product does not influence the corresponding discrete projection very much. Alternatively, one could interpret the result as a superconvergence result for the qualocation discretization of the operator $G$.

Suppose we want to solve $\phi$ from $G \phi=f$ by qualocation. We assume that $G$ is bounded above and below by positive numbers. Then the equation defining the discrete solution $\phi_{h}$ is

$$
\left(G \phi_{h}, \psi_{h}\right)_{h}=\left(f, \psi_{h}\right)_{h}, \quad \text { or equivalently, } \quad R_{h} G\left(\phi-\phi_{h}\right)=0 .
$$

The first formulation clearly shows a possible interpretation as projection in a weighted discrete inner product $G_{h}(\cdot, \cdot):=(G \cdot, \cdot)_{h}$, while the second reveals the qualocation orthogonality. Note that if $G$ is constant, $\phi_{h}=R_{h} \phi$. For smooth but not constant $G$, we will study the difference $\phi_{h}-R_{h} \phi$, assuming that there exists a solution $\phi_{h}$ of $(20)$.

Remark 3.1 Note that existence and uniqueness of a solution of (20) would follow immediately from the Lax-Milgram lemma if we would have proved explicitly that $G_{h}(\cdot, \cdot)$ defines an inner product on the discrete space, but we choose to follow a different line of proof. 
Theorem 3.2 There exists a number $h_{0}>0$ such that for all $0<h \leq h_{0}$, there exists a unique solution $\phi_{h} \in S_{h}$ of (20) that satisfies

$$
\left|\phi-\phi_{h}\right|_{h, p} \leq(1+C h)\left|\phi-R_{h} \phi\right|_{h, p} .
$$

Proof. Assume that for some given $\phi$, there exists a $\phi_{h}$ satisfying (20). Then, an easy manipulation, using (20) and $R_{h} \phi_{h}=\phi_{h}$, shows

$$
G\left(\phi_{h}-R_{h} \phi\right)=\left[R_{h}, G\right]\left(\phi_{h}-\phi\right) .
$$

This means that, for $g$ smooth enough, each of the commutator properties in Ths. 2.6 and 2.7 leads to

$$
\left\|\phi_{h}-R_{h} \phi\right\|_{p} \leq C h\left|\phi-\phi_{h}\right|_{h, p},
$$

where the constant $C$ depends on norms of derivatives of $g$ corresponding to which spline space was used to project upon. A simple triangle inequality, together with the equivalence of $\|\cdot\|_{p}$ and $|\cdot|_{h, p}$ on $S_{h}$, gives

$$
\begin{aligned}
\left\|\phi_{h}-R_{h} \phi\right\|_{p} & \leq C h\left|\phi-\phi_{h}\right|_{h, p} \\
& \leq C h\left|\phi-R_{h} \phi\right|_{h, p}+C h\left|\phi_{h}-R_{h} \phi\right|_{h, p} \\
& \leq C h\left|\phi-R_{h} \phi\right|_{h, p}+C h\left|\phi_{h}-R_{h} \phi\right|_{h, p},
\end{aligned}
$$

which, for all $h$ smaller than some $h_{0}>0$, results in

$$
\left\|\phi_{h}-R_{h} \phi\right\|_{p} \leq C h\left|\phi-R_{h} \phi\right|_{h, p} .
$$

Now, let $\phi=0$, then $R_{h} \phi=0$. Clearly, $\phi_{h}=0$ is a solution of (20). Let $\psi_{h}$ be any (possibly other) solution of (20). For this solution, the above analysis holds, resulting in $\psi_{h}=0$ for all $h \leq h_{0}$, concluded from (25). So, $\phi_{h}=0$ is the unique discrete solution belonging to $\phi=0$. By linearity, existence and uniqueness also follow for general $\phi_{h}$. For this $\phi_{h}$ we also find

$$
\begin{aligned}
\left|\phi-\phi_{h}\right|_{h, p} & \leq\left|\phi-R_{h} \phi\right|_{h, p}+\left|\phi_{h}-R_{h} \phi\right|_{h, p} \\
& \leq\left|\phi-R_{h} \phi\right|_{h, p}+C h|| \phi-\left.R_{h} \phi\right|_{h, p} . \\
& \leq\left|\phi-R_{h} \phi\right|_{h, p}+C h\left|\phi-R_{h} \phi\right|_{h, p} .
\end{aligned}
$$

This proves the theorem.

The above analysis shows that the presence of a variable coefficient results in a qualocation approximation that is only a higher order perturbation of the constant coefficient approximation. Or, put differently, if $\phi$ solves the variable coefficient equation, then its qualocation approximation does not differ much from the qualocation approximation of the constant coefficient equation with the right hand side adapted in such a way, that again $\phi$ is the exact solution. Or, again put differently, if we write $R_{h}^{g}$ for weighted discrete projection, we have found that

$$
\left\|\left(R_{h}^{g}-R_{h}\right) \phi\right\|_{p}=\left\|R_{h}\left(R_{h}^{g}-I\right) \phi\right\|_{p} \leq C h\left|\left(R_{h}^{g}-I\right) \phi\right|_{h, p} .
$$

Again, note that for $h$ small enough, we can replace the $R_{h}^{g}$ in the right-hand side by $R_{h}$ by using a triangle inequality and the equivalence of norms. 


\subsection{A variable coefficient operator equation}

A second application is of similar nature. We consider the difference between qualocation approximation in the weighted and the non-weighted version of the discrete inner product. Let $L: C(I) \rightarrow C(I)$ satisfy the following conditions,

$$
\forall \psi_{h} \in S_{h}^{0, r}, \quad\left|\left(L \psi_{h}, \psi_{h}\right)_{h}\right| \geq \beta\left|\psi_{h}\right|_{h, 2}^{2} \quad \text { and } \quad \forall \psi \in C(I), \quad|L \psi|_{h, 2} \leq M\|\psi\|_{2},
$$

where $\beta$ and $M$ are constants independent of $h$. In some situations, these properties can be directly derived from the corresponding non-discrete properties of coercivity and continuity. Then, for fixed exact solution $\phi$, consider the equations

$$
L \phi=f \text { and } \quad G L \phi=G f
$$

The respective qualocation approximations $\phi_{h}$ and $\phi_{h}^{g}$ in $S_{h}^{0, r}$ are uniquely defined by the qualocation orthogonality relations

$$
R_{h} L\left(\phi-\phi_{h}\right)=0 \text { and } R_{h}^{g} L\left(\phi-\phi_{h}^{g}\right)=0,
$$

as we will prove along the way, while comparing the two discrete solutions.

Proposition 3.3 For each $\phi \in C(I)$, there exists exactly one $\phi_{h} \in S_{h}^{0, r}$ such that $R_{h} L\left(\phi-\phi_{h}\right)=0$.

Proof. For $\phi=0$, there exists, obviously, at least one discrete solution $\phi_{h}=0$. Now suppose $\psi_{h}$ is any solution (for $\phi=0$ ). Then,

$$
\beta\left|\psi_{h}\right|_{h, 2}^{2} \leq\left|\left(L \psi_{h}, \psi_{h}\right)_{h}\right|=\left|\left(L \phi, \psi_{h}\right)_{h}\right|=0 .
$$

So, $\psi_{h}=0$. By linearity of the problem, this means that the discrete system matrix is invertible, hence existence and uniqueness follow.

Theorem 3.4 There exists a number $h_{0}>0$ such that for all $h \leq h_{0}$ and for each $\phi \in C(I)$ there exists exactly one $\phi_{h}^{g} \in S_{h}^{0, r}$ such that $R_{h}^{g} L\left(\phi-\phi_{h}^{g}\right)=0$. Moreover,

$$
\left\|\phi-\phi_{h}^{g}\right\|_{2} \leq(1+C h)\left\|\phi-\phi_{h}\right\|_{2} \text {. }
$$

Proof. Assume that for some given $\phi$ there exists a solution $\phi_{h}^{g}$. As in the previous section, we will compare the (or better, any) solution $\phi_{h}^{g}$ of the variable coefficient equation with the constant coefficient solution $\phi_{h}$. From the orthogonalities (30) it immediately follows that

$$
R_{h} L\left(\phi_{h}^{g}-\phi_{h}\right)=\left(R_{h}-R_{h}^{g}\right) L\left(\phi_{h}^{g}-\phi\right),
$$

which leads to

$$
\begin{aligned}
\beta\left|\phi_{h}^{g}-\phi_{h}\right|_{h, 2}^{2} \leq & \left|\left(L\left(\phi_{h}^{g}-\phi_{h}\right), \phi_{h}^{g}-\phi_{h}\right)_{h}\right|=\left|\left(R_{h} L\left(\phi_{h}^{g}-\phi_{h}\right), \phi_{h}^{g}-\phi_{h}\right)_{h}\right| \\
& \leq\left|\left(R_{h}-R_{h}^{g}\right) L\left(\phi_{h}^{g}-\phi\right)\right|_{h, 2}\left|\phi_{h}-\phi_{h}^{g}\right|_{h, 2} .
\end{aligned}
$$


Using the result (27) of the previous section together with 2-stability of $R_{h}$ and the orthogonality $R_{h}^{g} L\left(\phi_{h}^{g}-\phi\right)=0$, we conclude,

$$
\left|\phi_{h}-\phi_{h}^{g}\right|_{h, 2} \leq \frac{C}{\beta} h\left|L\left(\phi-\phi_{h}^{g}\right)\right|_{h, 2} \leq \frac{C M}{\beta} h\left\|\phi-\phi_{h}^{g}\right\|_{2} .
$$

Again, let $\phi=0$, then $\phi_{h}^{g}=0$ is a discrete solution. Also, for $\phi=0$ we have that $\phi_{h}=0$. For any discrete solution $\psi_{h}^{g}$ we conclude from (35) that (using the equivalence of norms on $S_{h}^{0}$ ),

$$
\left|\psi_{h}^{g}\right|_{h, 2} \leq C h \|\left.\psi_{h}^{g}\right|_{2} \leq C h\left|\psi_{h}^{g}\right|_{2}
$$

which means that, for $h$ small enough, $\phi_{h}^{g}=0$ is the unique solution. So, for $h$ small enough, the system matrix is invertible, and existence and uniqueness follows also for all other $\phi \in C(I)$. For those $\phi$ we also have

$$
\begin{aligned}
\left\|\phi-\phi_{h}^{g}\right\|_{2} & \leq\left\|\phi-\phi_{h}\right\|_{2}+\left\|\phi_{h}-\phi_{h}^{g}\right\|_{2} \\
& \leq\left\|\phi-\phi_{h}\right\|_{2}+C\left|\phi_{h}-\phi_{h}^{g}\right|_{h, 2} \\
& \leq\left\|\phi-\phi_{h}\right\|_{2}+\frac{C M}{\beta} h\left\|\phi-\phi_{h}\right\|_{2},
\end{aligned}
$$

where in the last step we used (35) with $\phi_{h}^{g}$ replaced by $\phi_{h}$, which is (with a different constant $C$ ) possible for $h$ small enough after a triangle inequality.

From this, we also conclude again that the presence of a variable coefficient can be dealt with by means of the commutator property. Existence and uniqueness as well as stability and convergence follow from the constant coefficient case.

Remark 3.5 In the paper [10], the commutator property for smoothest splines is used to prove stability and convergence, and even superconvergence, for qualocation methods for an operator that is the sum of an even and an odd pseudo-differential operator, both with variable coefficients. Because of the very technical character of that paper we will not include details here, but once again stress the practical importance of the commutator property.

\section{Numerical experiments}

We now test the results so far by running some numerical experiments. We set up a qualocation method on the circle using continuous piecewise linear approximations on uniform grids (the first three experiments) and on a non-uniform grid (fourth experiment) and compare the projections $R_{h}$ and $R_{h}^{g}$ for the weight-function $g$ and exact solution $\phi$ given by (see Figure 2)

$$
g(x)=1+\exp (\sin (2 \pi x)) \sin ^{2}(2 \pi(x+0.1)), \quad \text { and } \quad \phi(x)=\cos ^{2}\left(2 \pi \sin \left(\frac{1}{2} \pi x\right)\right) / g(x) .
$$

In the first experiment, we took the interpolatory rule $\xi_{1}=0$ and $\xi_{2}=1$ with both weights $w_{1}=w_{2}=0.5$. In the second experiment, we used the $p$-stable one-point rule $\xi=0.4$ with weight $w=1$. Finally, in the third and fourth experiment, we took 
the $p$-stable rule $\xi_{1}=1 / \pi, \xi_{2}=1 / \sqrt{2}, \xi_{3}=1$ with weights $w_{1}=\sqrt{2}, w_{2}=\pi, w_{3}=1$.
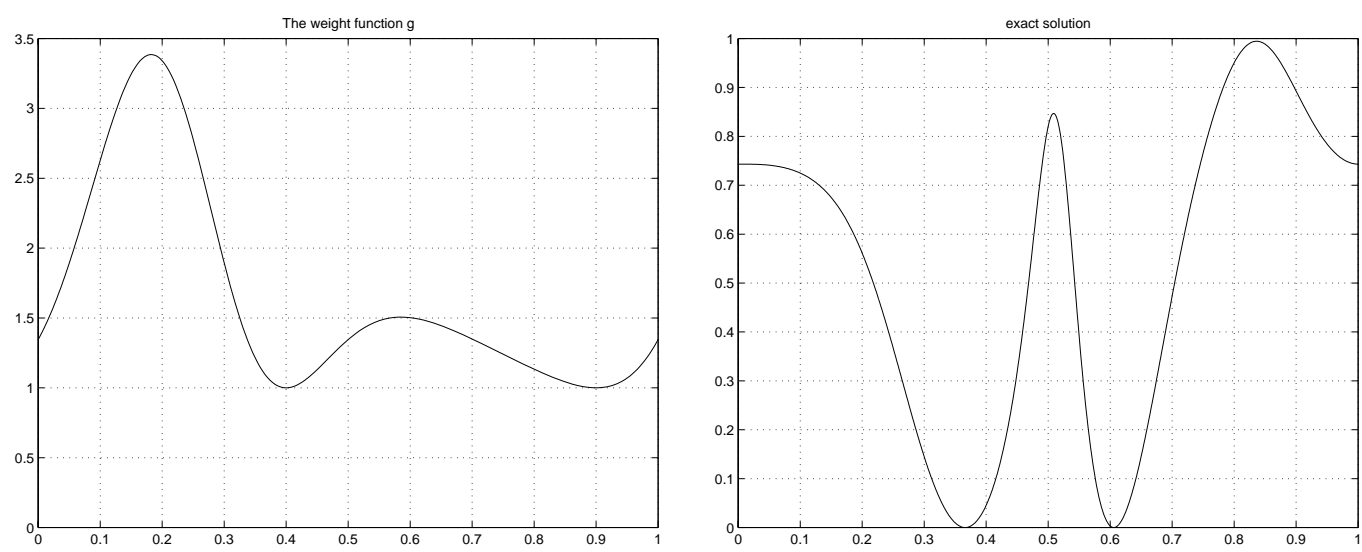

Figure 2. The weight function $g$ (left) and the exact solution $\phi$ (right).

The results are summarized in the following tables, in which we present (from left to right) the number of intervals, the exact error $\left\|\phi-R_{h} \phi\right\|_{2}$, the reduction of those errors, the difference $\left\|\left(R_{h}^{g}-R_{h}\right) \phi\right\|_{\infty}$ and the reduction of these differences.

\section{First experiment}

As expected, we see that for the interpolatory projection, there is no difference between the projections $R_{h}$ and $R_{h}^{g}$. This follows immediately from the fact that the nodal basis is also orthogonal with respect to the weighted inner product. One could conclude the same by noticing from (22) that the spline $\phi_{h}-R_{h} \phi$ must have two zeros per sub-interval.

\begin{tabular}{|r||l|l|c|c|}
\hline$h^{-1}$ & $\left\|\phi-R_{h} \phi\right\|_{2}$ & red. error & $\left\|\left(R_{h}^{g}-R_{h}\right) \phi\right\|_{\infty}$ & red. supcv. \\
\hline \hline 4 & $2.9715 e-001$ & $\cdots$ & 0 & $\cdots$ \\
\hline 8 & $5.4673 e-002$ & $5.4350 e+000$ & 0 & $\cdots$ \\
\hline 16 & $2.3205 e-002$ & $2.3561 e+000$ & 0 & $\cdots$ \\
\hline 32 & $6.4725 e-003$ & $3.5852 e+000$ & 0 & $\cdots$ \\
\hline 64 & $1.6399 e-003$ & $3.9469 e+000$ & 0 & $\cdots$ \\
\hline 128 & $4.1134 e-004$ & $3.9868 e+000$ & 0 & $\cdots$ \\
\hline 256 & $1.0292 e-004$ & $3.9967 e+000$ & 0 & $\cdots$ \\
\hline
\end{tabular}

\section{Second experiment}

\begin{tabular}{|r||l|l|r|l|}
\hline$h^{-1}$ & $\left\|\phi-R_{h} \phi\right\|_{2}$ & red. error & $\left\|\left(R_{h}^{g}-R_{h}\right) \phi\right\|_{\infty}$ & red. supcv. \\
\hline \hline 4 & $3.7306 e-001$ & $\cdots$ & $2.0338 e-002$ & $\cdots$ \\
\hline 8 & $3.4907 e-002$ & $1.0687 e+001$ & $1.2792 e-002$ & $1.5898 e+000$ \\
\hline 16 & $1.6379 e-002$ & $2.1313 e+000$ & $2.9543 e-003$ & $4.3301 e+000$ \\
\hline 32 & $3.6650 e-003$ & $4.4689 e+000$ & $4.4491 e-004$ & $6.6401 e+000$ \\
\hline 64 & $7.9783 e-004$ & $4.5937 e+000$ & $5.8508 e-005$ & $7.6042 e+000$ \\
\hline 128 & $1.8648 e-004$ & $4.2783 e+000$ & $7.3815 e-006$ & $7.9264 e+000$ \\
\hline 256 & $4.5097 e-005$ & $4.1351 e+000$ & $9.2232 e-007$ & $8.0032 e+000$ \\
\hline
\end{tabular}


In the above tabular we see that the two projections are not equal anymore. Also, the difference between the two is clearly of the expected higher order $h^{3}$.

\section{Third experiment}

Also the results from the "nonsense" (though $p$-stable) quadrature rule

$$
\int_{0}^{1} f(x) d x \approx \sqrt{2} f\left(\pi^{-1}\right)+\pi f(1 / \sqrt{2})+f(1)
$$

are according to the theory. Optimal order convergence for the projections, and supercloseness between the two different projections themselves clearly show.

\begin{tabular}{|r||l|l|r|l|}
\hline$h^{-1}$ & $\left\|\phi-R_{h} \phi\right\|_{2}$ & red. error & $\left\|\left(R_{h}^{g}-R_{h}\right) \phi\right\|_{\infty}$ & red. supcv. \\
\hline \hline 4 & $3.2632 e-001$ & $\cdots$ & $1.4799 e-001$ & $\cdots$ \\
\hline 8 & $5.1389 e-002$ & $6.3501 e+000$ & $4.5285 e-002$ & $3.2679 e+000$ \\
\hline 16 & $1.5543 e-002$ & $3.3063 e+000$ & $1.3705 e-002$ & $3.3044 e+000$ \\
\hline 32 & $3.3991 e-003$ & $4.5727 e+000$ & $2.1003 e-003$ & $6.5250 e+000$ \\
\hline 64 & $7.5254 e-004$ & $4.5168 e+000$ & $2.7336 e-004$ & $7.6834 e+000$ \\
\hline 128 & $1.7769 e-004$ & $4.2351 e+000$ & $3.4648 e-005$ & $7.8895 e+000$ \\
\hline 256 & $4.3241 e-005$ & $4.1094 e+000$ & $4.3567 e-006$ & $7.9529 e+000$ \\
\hline
\end{tabular}

\section{Fourth experiment}

Finally, we tested the theory with respect to the effects of non-uniform meshes. Typically, the following behavior could be observed. We defined, from a uniform mesh $\pi_{h}$ a non-uniform mesh $z\left(\pi_{h}\right)$ with $z(x)=\sin ^{t}\left(\frac{1}{2} \pi x\right)$ for some value of $t$. For the quadrature rule of the third experiment, and with $t=4$, we obtained the following results. Asymptotically, our expectations are confirmed.

\begin{tabular}{|r||l|l|r|l|}
\hline$h^{-1}$ & $\left\|\phi-R_{h} \phi\right\|_{2}$ & red. error & $\left\|\left(R_{h}^{g}-R_{h}\right) \phi\right\|_{\infty}$ & red. supcv. \\
\hline \hline 4 & $2.8655 e-01$ & 0 & $1.1330 e-01$ & 0 \\
\hline 8 & $2.1172 e-01$ & $1.3535 e+00$ & $1.3823 e-01$ & $8.1967 e-01$ \\
\hline 16 & $3.2044 e-02$ & $6.6070 e+00$ & $8.2309 e-03$ & $1.6794 e+01$ \\
\hline 32 & $1.2203 e-02$ & $2.6259 e+00$ & $1.7638 e-03$ & $4.6665 e+00$ \\
\hline 64 & $2.3322 e-03$ & $5.2324 e+00$ & $1.8058 e-04$ & $9.7675 e+00$ \\
\hline 128 & $5.4762 e-04$ & $4.2589 e+00$ & $6.5817 e-06$ & $2.7437 e+01$ \\
\hline 256 & $1.3482 e-04$ & $4.0619 e+00$ & $3.7351 e-07$ & $1.7621 e+01$ \\
\hline 512 & $3.3576 e-05$ & $4.0153 e+00$ & $2.2797 e-08$ & $1.6384 e+01$ \\
\hline
\end{tabular}

\section{$5 \quad$ Further topics of interest}

There is a large number of unsolved problems in the setting of discrete projections. Even though theory has been developed for derivatives (discrete $H^{1}$ results) in [5], a smooth theory for fully discrete Galerkin methods for two-point boundary value problems is still missing. This is mainly due to the lack of an equivalent of "integration by parts", i.e., symbolically,

$$
(D F, G)_{h} \neq\left. F G\right|_{0} ^{1}-(F, D G)_{h},
$$


not even for $F$ and $G$ in the spline space, unless the integration rule has a certain accuracy (which is what we did not want to assume in the first place). It seems logical that in a context of numerical integration, a corresponding numerical differentiation operator needs to be defined which has the desired properties.

An even more important extension of the theory would be the extension to two space dimensions. Discrete orthogonal projection theory for tensor product spaces does not seem to be a real problem, but it is unclear what to do in the simplectic case. Research is in progress.

\section{Acknowledgments}

The author gratefully acknowledges the support of a Research Fellowship of the Royal Netherlands Academy of Arts and Sciences.

\section{References}

[1] C. de Boor, $A$ bound on the $L_{\infty}$-norm of $L_{2}$-approximation by splines in term of a global mesh ratio, Math. Comp. 30, pp. 765-771, 1976.

[2] M. Crouzeix and V. Thomée, The stability in $L_{p}$ and $W_{p}^{1}$ of the $L_{2}$-projection onto finite element function spaces, Math. Comp. 48, pp. 521-532, 1987.

[3] G.A. Chandler and I.H. Sloan, Spline qualocation methods for boundary integral equations, Numer. Math. 58, pp. 537-567, 1990.

[4] R.D. Grigorieff and I.H. Sloan, Stability of discrete orthogonal projections for continuous splines, Bull. Austral. Math. Soc. 58, pp. 307-332, 1998.

[5] R.D. Grigorieff, I.H. Sloan and J.H. Brandts Superapproximation and commutator properties of discrete orthogonal projections for continuous splines, Submitted, 2000.

[6] W. McLean and S. Prössdorf, Boundary element collocation methods using splines with multiple knots, Numer. Math. 74, pp. 419-451, 1996.

[7] S. Prössdorf, Ein Lokalisierungsprinzip in der Theorie der Spline-Approximationen und einige Anwendungen, Math. Nachr. 119, pp. 239-255, 1984.

[8] I.H. Sloan and W. Wendland, Qualocation methods for elliptic boundary integral equations, Numer. Math. 79, pp. 451-483, 1998.

[9] I.H. Sloan and W. Wendland, Commutator properties for periodic splines, J. Approx. Theory, 97, pp. 254-281, 1999.

[10] I.H. Sloan and W. Wendland, Spline qualocation methods for variable-coefficient elliptic equations on curves, Institut Mittag-Leffler, Report No. 5, 1997/1998, ISSN 1103-467X, Numer. Math., to appear.

[11] L. Wahlbin, Superconvergence in Galerkin finite element methods, Lecture Notes in Mathematics 1605, Springer, Berlin, 1995. 